\title{
Colloid Monolayers as Versatile Lithographic Masks
}

\author{
Frank Burmeister,* Claudia Schäfle, Thomas Matthes, Matthias Böhmisch, \\ J ohannes Boneberg, and Paul Leiderer \\ Department of Physics, University of Konstanz, P.O. Box 5560 M676, \\ D-78434 Konstanz, Germany
}

Received December 17, 1996. In Final Form: March 7, $1997^{\circledR}$

\begin{abstract}
Hexagonally closed packed monolayers of colloids have found more and more applications, e.g. as lithographic masks. The monolayers are usually produced with the help of a self-organizing process where a suspension of colloids is applied to the desired substrate and left to dry. This method requires a good wettability and smoothness of the substrate, which severely limits the number of possi ble substrates. We present a new method for theapplication of coll oid monol ayers to al most any surface wher ethesedifficulties are circumvented. At first the monolayers are fabricated on gl ass substrates and afterwards floated off on a water surface. From there, they aretransferred tothedesired substrate. Examples wheretransferred monolayers wereused as lithographic masks are shown on glass, indium tin oxide, and tungsten diselenide. The transfer of a colloid monolayer to a copper grid for transmission electron microscopy demonstrates the applicability of the technique to curved surfaces as well.
\end{abstract}

\section{Introduction}

The fabrication of closed packed monolayers of submicron-sized spherical colloids (mainly polystyrene latices) is now well established and widely used in various fields of research.

On one hand, 2D colloidal crystals are of fundamental interest and have been studied e.g. as model systems for melting in two dimensions. ${ }^{1}$ More application-oriented were other experiments, where the colloidal arrays have been utilized either as lithographic or etching masks to structuresurfaces on a scal esmaller than thewavel ength of visible light. ${ }^{2,3}$ Further applications of this technique are e.g. the production of tiny ellipsoidal silver particles for optical absorbance experiments ${ }^{4}$ or the fabrication of a phonon diffraction grating. ${ }^{5}$

At the same time, the nucl eation of $2 \mathrm{D}$ crystals as well as the driving forces for the crystallization process has been investigated both experimentally and theoretically. ${ }^{6-12}$ These studies in turn allowed the development of new and relatively simplemethods for the production of colloid monolayers, with particle diameters ranging from $40 \mathrm{~nm}$ to several microns with a size of the polycrystalline monolayers up toseveral $\mathrm{cm}^{2} .13,14$ Thesemethods usually involved an application of a colloid suspension to a solid substrate and a controlled evaporation of the suspension.

${ }^{\otimes}$ Abstract published in Advance ACS Abstracts, May 1, 1997. 1200.

(1) Murray, C. A.; van Winkle, D. J . Phys. Rev. Lett. 1987, 58 (12),

(2) Fischer, U. Ch.; Zingsheim, H. P. J . Vac. Sci. Technol. 1981, 19 (4), 881.

(3) Deckman, H. W.; Dunsmuir, J . H. Appl. Phys. Lett. 1982, 41 (4), 377.

(4) Buncick, M. C.; Wormack, R. J .; Ferell, T. L. J . Opt. Soc. Am. B 1987, 4 (6), 927.

(5) Vu, P. D.; Olson, J. R.; Pohl, R. O. Ann. Phys. 1995, 4, 9.

(6) Kralchevsky, P. A.; Paunov, V. N.; I vanov, I. B.; Nagayama, K. J . Colloid Interface Sci. 1992, 151 (1), 79.

(7) Denkov, N. D.; Velev, O. D.; Kralchesky, P. A.; Ivanov, I. B.; Yoshimura, H.; Nagayama, K. Langmuir 1992, 8, 3183.

(8) Denkov, N. D.; Velev, O. D.; Kralchesky, P. A.; Ivanov, I. B.; Yoshimura, H.; Nagayama, K. Nature 1993, 361, 26.

(9) Dushkin, C. D.; Y oshimura, H.; Nagayama, K. Chem. Phys. Lett. 1993, $204(5,6), 455$.

(10) Kralchevsky, P. A.; Nagayama, K. Langmuir 1994, 10, 23.

(11) Dimitrov, A. S.; Dushkin, C. D.; Yoshimura, H.; Nagayama, K. Langmuir 1994, 10, 432.

(12) Adachi, E.; Dimitrov, A. S.; Nagayama, K. Langmuir 1995, 11, 1057.

(13) Dimitrov, A. S.; Nagayama, K. Langmuir 1996, 12, 1303.

(14) Micheletto, R.; Fukuda, H.; Ohtsu, M. Langmuir 1995, 11, 3333.
Nevertheless, regarding all the experiments done in this way, one immediately sees a major restriction in the applicability of colloidal masks for nanostructuring of surfaces. When producing the monolayers, all authors point out several requirements for thesubstratethey used for the crystallization process: (a) chemical homogenity of the surface, (b) flatness of the substrate, i.e. the mean surface corrugation should be much smaller than the particle diameter, and (c) a clean and very hydrophilic surface (the colloids were al ways suspended in water). As a consequence, al most all experiments have been done on glass plates or polished silicon wafers as substrates. ${ }^{5,7,12,14}$ Mica was also used in a few occasions, ${ }^{9,11}$ whereas other materials, e.g. semi conductors or metals, havehardly been used at all. To overcome these difficulties, the colloid monolayers could beassembled alternatively on a liquidliquid ${ }^{15}$ or liquid-gas ${ }^{16}$ interface. From there, the monolayer could be transferred in principle to any substrate, either by the subphase-lowering method ${ }^{17}$ or by the Langmuir-Blodgett technique. These methods still re quire a special cell design and/or a precise control of surfactant concentration and $\mathrm{pH}$. The quality of the obtained crystals tends to beslightly worsewhen compared to that of crystals obtained on solid substrates.

Instead, we want to present a new method to apply colloidal masks for structuring surfaces which combines the advantages of a very simple preparation and an applicability to almost any kind of surface. In a first step, we prepared colloid monolayers on glass plates as will be described below. Next, we transferred the monolayer arrays as a whole from the glass plates onto the desired substrate in a kind of inverse Langmuir-Blodgett technique. The monolayers could then be used as a lithographic mask. In this way, we did not need any wetting agents or surfactants which might form residuals on the surface.

The materials and the details of the two-step procedure we have developed will be described in the first section. In the second section, we present some experimental results we obtained on three different substrates. They will be discussed in the following section.

(15) Lazarov, G. S.; Denkov, N. D.; Velev, O. D.; Kralchesky, P. A J. Chem. Soc., Faraday Trans. 1994, 90 (14), 2077.

(16) Fulda, K.-U.; Ticke, B. Adv. Mater. 1994, 6 (4), 288.

(17) I wahashi, M.; Kikuchi, K.; Achiba, I.; Ikemoto, I.; Araki, T.; Mochida, T.; Yokoi, S.-I.; Tanaka, A.; I riyama, K. Langmuir 1992, 8 , 2980. 


\section{Experimental Section}

The sulfatized and sterically stabilized polystyrene latex spheres were purchased from Bang Laboratories, Inc. with a mean diameter of $840 \mathrm{~nm}$, measured by static light scattering. The initial volume fraction of $10 \%$ was diluted with millipore water to about $1 \%$

Monolayer Fabrication. In principle, we followed the method of Micheletto et al. ${ }^{14}$ for the production of 2D arrays of colloids in a first step. Microscope glass slides were cut into pieces of roughly $1 \mathrm{~cm}^{2}$ and ultrasonicated in mucasol (Merz $\mathrm{GmbH}$, Frankfurt), acetone, ethanol, and deionized water prior to use. Then, a drop of $10-20 \mu \mathrm{L}$ of diluted colloidal suspension was applied to the slightly tilted substrate. The evaporation of the excess water took place in a cl osed chamber with control over temperature and ambient humidity. The evaporation process lasted several hours, leaving close-packed polycrystalline monolayers of up to $1 \mathrm{~cm}^{2}$, with typical domain sizes of several hundreds of square micrometers.

Transfer of Monolayers. The crucial step in our method is the removal of the colloid monolayer as a whole from the glass substrate on which it was prepared. This was done by floating off the whole monolayer in millipore water, which simply means a controlled dipping of the glass plate into water at a small but constant velocity. As soon as the glass plate touched the water surface, water started to penetrate the gap between the colloid monolayer and the glass surface. In this way, the monolayer was stripped off gradually and remained at the water surface without any wrinkling or folding. From there, it was transferred to the desired substrate by touching it from above with the horizontally held substrate.

In order to performa controlled dipping of theglass substrates, we used an apparatus which was originally built for sol-gel processes. With itshelp, wecould movetheglass plates vertically into a water-containing vessel at constant vel ocities smaller than $1 \mathrm{~mm} / \mathrm{s}$. This turned out to be crucial for successfully floating off the monolayers. Afterward we could still observe the typical irridescent colors ${ }^{9,11}$ from the crystalline arrays floating on the water surface. This was a dear indication of their preserved ordering. After some practice, we managed to transfer polycrystalline arrays as large as $0.5 \mathrm{~cm}^{2}$.

Materials and Instrumentation. We used three different materials as substrates in the final step of our method. Thefirst ones were glass plates coated with $100 \mathrm{~nm}$ of ITO (indium tin oxide). ITO is transparent and sufficiently conductive to be a suitable substrate for both optical and el ectron microscopy. The second kind of samples were single crystals of the layered semiconductor $\mathrm{WSe}_{2}$, which were grown by the vapor phase transport method. ${ }^{18}$ F or a review of its material properties see for instance ref 19. After cleavage with an adhesive tape, they exhibited atomically flat and inert van der Waals surfaces. The third substrates were copper grids with a mean mesh size of 10 $\mu \mathrm{m}$. They are normally used for electron microscopy and were purchased from Plano W. Plannet GmbH, Wetzlar.

For a first inspection of the monolayer arrays, we used an optical microscope with a magnification up to $1000 \times$, which was equipped with a CCD camera and a videoprinter.

Metal-coated or conductive samples were also examined with a Stereoscan 100 SEM (scanning electron microscope) from Cambridgel nstruments. N onconductivesamples were sputtercoated with gold before examination. The AFM (atomic force microscope) measurements were carried out with a Topometrix Explorer in the constant force mode. Weutilized V-shaped $\mathrm{Si}_{3} \mathrm{~N}_{4}$ cantilevers with nominal force constants of $0.032 \mathrm{~N} / \mathrm{m}$. The tip shape was pyramidal with a quadratic base. For the STM (scanning tunnelingmicroscope) measurements, weused a homebuilt instrument in the constant current mode with electrochemically etched Pt/lr tips.

\section{Results}

To demonstrate the basic principles of our technique, we show a colloid monolayer which was transferred onto an ITO-coated glass plate. The ITO substrate was not

(18) Al-Hilly, A.; Evans, B. J . Cryst. Growth 1972, 15, 93.

(19) Aruchamy, A. Photod ectrochemistryand Photovol taics of Layered Semiconductors; Kluwer: London, New York, 1992.

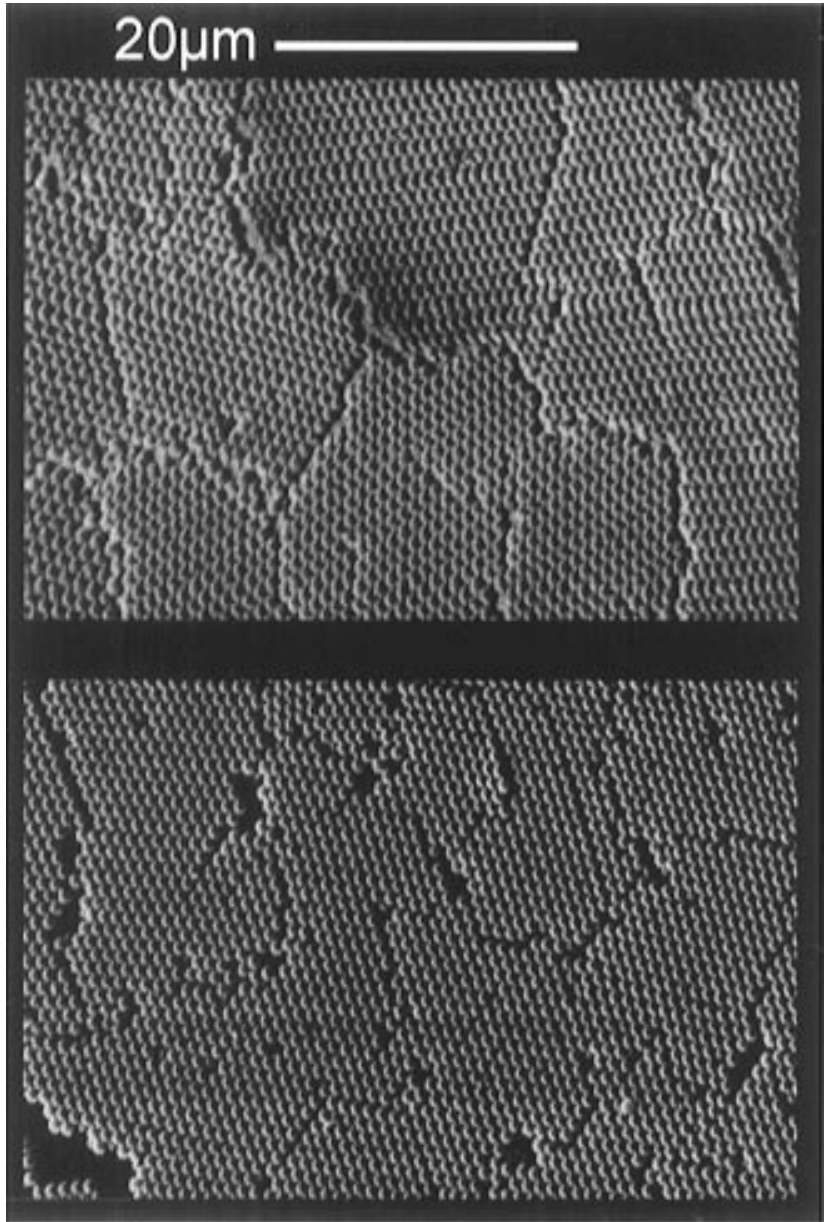

Figure 1. SEM micrographs of gold-coated coll oi d monolayers (diameter $840 \mathrm{~nm}$ ), magnification $2300 \times$ : (a) on glass, as prepared; (b) transferred onto ITO.

pretreated prior to use and exhibited a mean surface corrugation of $20 \mathrm{~nm}$ as measured by AFM. Before the transfer, we evaporated a thin layer of approximately 60 $\mathrm{nm}$ of gol d ontothecolloid monolayer, which was fabricated as described above.

Figure 1 shows a SEM comparison between the colloid monolayer before the transfer (a), where it is still on the glass substrate, and (b) after the transfer on the ITO substrate. In both cases, the typical structure of monolayer arrays with differently oriented crystal domains, cracks, ${ }^{20}$ and grain boundaries can be seen. In the transferred monolayer, the indi vidual crack size appears to be larger than before the transfer. Also, at some locations, single particles are missing. However, the suitability as a lithographic mask is still given and demonstrated by the AFM images Figure 2a. They were both taken with thesametip immediately after each other.

In Figure 2a, the glass substrate after floating off the colloid monolayer can be seen. Regularly arranged gold dots are left on the surface. Their symmetry and arrangement mirrors the former positions of the voids between the spheres. Some larger gold patches are due to stacking faults and cracks in the colloid monolayer. After floating off the monolayer, it was picked up with the ITO substrateand used as a lithographicmask for a second time. After the second deposition of $60 \mathrm{~nm}$ of $\mathrm{Au}$, the colloids were removed in an ultrasonic bath. The result can be seen in Figure 2b. Note that the gold dots are

(20) Crack formation occurs dueto swelling of the colloids when they are suspended in water and a consequent shrinking after drying and transfer to the high-vacuum conditions in a SEM. 


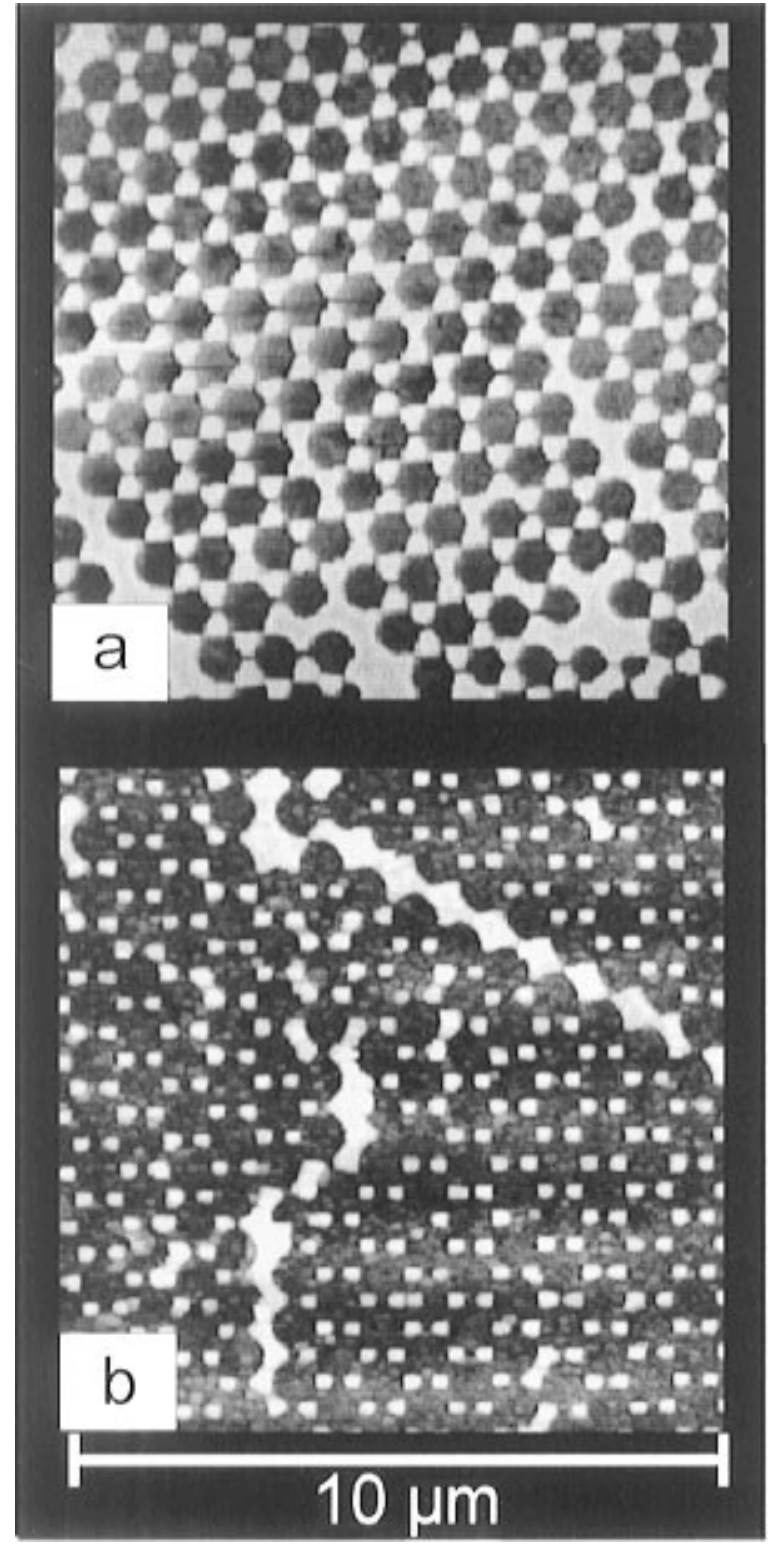

Figure 2. (a) AFM micrographs of gold nanostructures evaporated through the colloid monolayer of Figure 1: (a) on glass; (b) on ITO. The depicted area is $10 \mu \mathrm{m}^{2}$. The height of the structures is $60 \mathrm{~nm}$.

smaller than the structures in Figure 2a. Their slightly reduced size can be explained by the decreased size of the voids duetothefirst gold coating. In both cases, theaspect ratios of the gold dots were comparable to those of the imaging tip, which resulted in tip convolution effects. As a consequence, the gold dots did not appear triangular as would be expected. However, their trigonal shape was later confirmed in SEM measurements.

Wethen turned toa semiconducting substrateand chose a $\mathrm{WSe}_{2}$ single crystal. Because its surface is extremly hydrophobic, all previous attempts to fabricate a colloid monolayer on this surfacefailed. With our new technique however, wesucceeded in transferring a colloid monolayer of several square millimeters onto the crystal. The monol ayer wasthen used as a lithographic mask as before. Prior to a final examination by an STM (Figure 3), we removed the particles with milli porewater in an ultrasonic bath.

As expected, we observed hexagonal patterns of gold dots on the surface with their height corresponding to the evaporated thickness of the gold film. Again, their shape and lateral dimensions could not be exactly identified, as

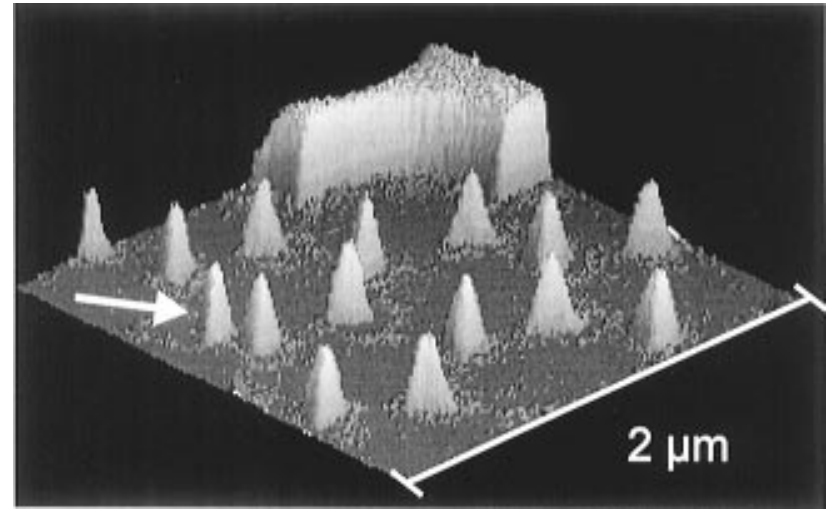

Figure 3. STM micrograph of gold nanostructures on the surface of a WSe single crystal. The picture is represented in a 3D mode; the height of the structures is $70 \mathrm{~nm}$. The arrow highlights a metal dot which shifted sideways and left its lattice site.

their dimensions and aspect ratios were comparable to those of the STM tip (a SE M micrograph taken afterward revealed triangular structures with a sidelength of roughly $150 \mathrm{~nm}$ ). One of the gold dots, which is marked by an arrow, moved out of its lattice site, probably during the removal of the colloids in theultrasonic bath. This might possibly be due to the high surface mobilities of metal islands on such surfaces. ${ }^{21}$ In the vicinity of the large gold dots, smaller hillocks with diameters of several nanometers could be observed. Presumably, gol d atoms diffused along the surface during the deposition and clustered into these hillocks. It is also remarkable that the surrounding $\mathrm{WS}_{2}$ surface was still atomically smooth and could be imaged with atomic resolution (only visible at higher magnifications). There, it was even possibleto obtain I-V curves which exhibited Schottky-likebehavior similar to I-V curves measured on freshly cleaved samples.

To our knowledge, this is the first time that regularly arranged metal dots of submicron size could be deposited and imaged by STM on such a surface. I-V spectroscopy with local resolution should now bepossible to investigate e.g. the local band-bending around these metal islands.

As a last example, weused a 'substrate' which is neither hydrophilic nor flat: a copper grid, normally used for transmission electron microscopy. Again, the monolayer was picked up by touching it from above with the copper grid. Parts $a$ and $b$ of Figure 4 are photographs of optical microscope pictures, taken in the reflection and transmission modes, respectively. Great portions of the grid were covered with a monolayer, even in the openings between the copper bars. Because it was not possible to focus the colloids on a copper bar and those in the center of the mesh next to it simultaneously (focus depth approximately $0.5 \mu \mathrm{m})$, we concluded a slight bending of the monolayer. This was again a clear indication of the unexpected stability of the colloid monolayers and demonstrates that our method should be applicable to curved surfaces as well. Finally in Figure 4c, which wastaken with theSEM, one sees the same monolayer imaged from the backside; i.e., thegrid was rotated for inspection. In fact, that means that wehavefabricated a transportablelithographicmask.

\section{Discussion}

Floating off the colloid monolayers was strongly based on the unique properties of water with its high surface 

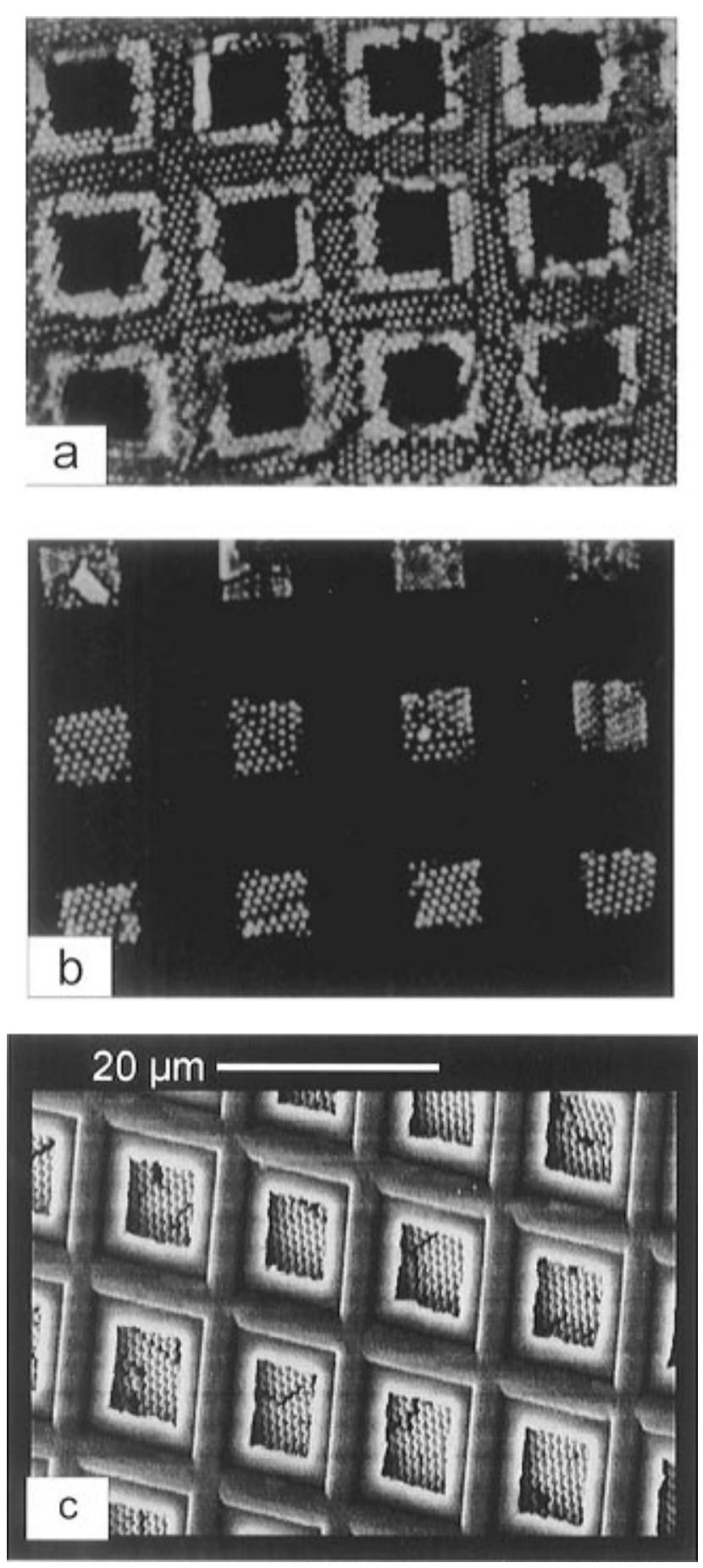

Figure 4. Optical microscope pictures of a TEM grid covered by a transferred colloid monolayer: (a) reflection mode; (b) transmission mode. (c) SE M micrograph where the backside of thegrid of parts $a$ and $b$ can beseen. Notethepreserved ordering of the PS latices.

tension 22,23 and the difference in wettability between the glass substrates and the colloid monolayer. For instance, we managed to float off and pick up the same monolayer from a gl ass plate several times, while fl oating off colloid monolayers from other substrates than glass failed. Floating off the monolayers in other liquids than water was not successful either.

In a first approximation, we assume that, as soon as water penetrates the gap between the hydrophilic glass

(22) Israel achvili, J . N. I ntermol ecular and SurfaceF orces; Academic Press: New York, 1992; Chapter 8.

(23) Franks, F. Water: A Comprehensive Treatise; Plenum: New York, 1972-82; Vols. 1-7. surface and the mainly hydrophobic ${ }^{24,25}$ latex spheres, it forms rel atively large contact angle ${ }^{26}$ with the spheres. In this way, the particles are strongly attached to the water-air interface. 27,28 When thethickness of the water layer isfurther increased, thepartides remain at thewater surface and are thus detached from the substrate.

Another question which immediately arises is theorigin of the stability of the monolayers. It is well-known that the particles experience a mutual attraction due to van der Waals forces, which diverges at very small distances. In order to prevent coagulation, the particles are usually protected by a special surface treatment to induce electrostatic repulsion.24,25 This effectively leads to a potential barrier, which is much higher than their thermal energy kT. However, at theend of theevaporation process, when the top of the particles protrudes from the water surface, the particles experience strong capillary forces, which gives risetotheir aggregation. ${ }^{6}$ Furthermore, when the suspension had dried completely, the particles probably lost their el ectrostatic repulsion due to dissociated surface groups. ${ }^{15}$

As the surfactant concentration was not specified by the manufacturer, we also performed experiments with particles which were thoroughly cleaned prior to use in an ion exchanger. Neither the process of crystallization nor their behavior when they werefloated off showed any significant deviations compared to that of the uncleaned particles.

An aditional stabilization mechanism might arisefrom capillary forces between the colloid particles. As soon as the particle array is immersed into water, the water will form micromenisci in the array voids. The resulting surface tension force posseses a horizontal component pointing from the center of thespheres toward the voids. ${ }^{27}$

All in all, the particles weresostrongly bound that even a repeated immersion in water did not lead tothecomplete dissolution of the monolayers. In most cases, they broke into pieces, probably due to mechanical stresses during the floating process. These pieces were comparable to the '2D-sheets' observed by Velev et al. ${ }^{24}$ and consisted of typically 10-100 particles. Only on a few occasions were the monolayers so stable that they could be floated off without falling apart.

As a consequence, we usually heated the sample to 90 ${ }^{\circ} \mathrm{C}$ for $10 \mathrm{~min}$ or al ternatively evaporated a thin metal film. Either of thetwotreatments allowed a reproducible detachment of the colloid monolayer from the glass substrate.

However, theexistence of other binding mechanisms of the colloi ds than van der Waals forces, e.g. interdiffusion of polymer chains, cannot be ruled out completely by our experiments. Further investigations with combinations of different particles (e.g. silica particles) and surfactant concentrations would help to clarify this point. ${ }^{29}$

When compared to a normal polymer film of the same thickness, the colloid monolayer still consists of single particles with a maximum of six binding sites to their neighbors. Besides the el asticity of the polymer chains

(24) Dhont, J . K. G. An Introduction to the Dynamics of Colloids Elsevier: Amsterdam, 1996.

(25) MicrosphereSelection Guide; Bangs Laboratories, Inc.: Carmel, September 1995

(26) Hadjiiski, A.; Dimova, R.; Denkov, N. D.; Ivanov, I. B.; Borwankar, R. Langmuir 1996, 12, 6665.

(27) (a) Ivanov, I. B.; Kralchevsky, P. A.; Nikolov, A. D. J . Colloid Interface Sci. 1986, 112, 97. (b) Leenaars, A. F. M. In Particles on Surfaces; Mittal, K. L., Ed.; Plenum Press: New York, 1988; p 361. 2374.

(29) Very recent experimental results obtained with surfactant-free $300 \mathrm{~nm}$ PS-latices were al so comparable to those obtained with the 840 $\mathrm{nm}$ PS-latices. 
itself, this might bea reason for their observed flexibility, which is absolutely necessary for their applicability as lithographic masks: for simple geometrical reasons, the voids between the colloids must not be further away from the surface than a few microns. Otherwise, due to the finite extent of the evaporation source, structures originating from neighboring voids would overlap. I n our case, there exists a strong van der Waals attraction between thefloated colloidmonolayer and thesubstrate. Therefore, the monolayer tends to follow the corrugation of the substrate, thus maintaining a constant distance between the voids in thecolloid monolayer and thesubstratesurface which is on the order of the radius of the spheres. This may restrict the application of colloid monolayers, which have been floated off on relatively stiff el ectron mi croscopy grids, as transportable lithographic masks. Further experiments concerning this point are in progress.

\section{Conclusions}

We presented a novel parallel method for producing nanometer structures on arbitrary surfaces which leaves a surface al most free of contaminants and is easy to apply.
Hexagonally or dered monolayers of colloid particles were fabricated on glass and floated off on a water surface. The lithographic masks produced in this manner could be applied to any surface and also to a very hydrophobic (WSe $)$ and even curved surface. Using theselithographic masks, the side lengths of the resulting structures were on the order of $200 \mathrm{~nm}$. H owever, these structures would be smaller by at least a factor of ten ${ }^{14}$ if one employed smaller particles for themonolayer production. This opens up a new and interesting way to produce 'real' nanostructures or even quantum dots on surfaces. They could beindividually studied by scanning probe methods, where a distortion in their hexagonal arrangement duetolattice imperfections in thecolloid monolayer is of noimportance.

Acknowledgment. Wegratefully acknowledgehel pful discussions with D. Reim and S. Neser concerning the fabrication of the colloid monolayers. This work was financially supported by the Deutsche Forschungsgemeinschaft (DFG), SFB 513. 